\title{
ENERGY-EFFICIENT 3D UAV TRAJECTORY DESIGN FOR DATA COLLECTION IN WIRELESS SENSOR NETWORKS
}

\author{
Daniel Bonilla Licea*, Edmond Nurellari ${ }^{\dagger}$, and Mounir Ghogho* $\ddagger$ \\ * International University of Rabat, TICLab, Morocco \\ $†$ School of Engineering, University of Lincoln, UK \\ $\ddagger$ School of Electronic and Electrical Engineering, University of Leeds, UK \\ daniel.bonilla-licea@uir.ac.ma, enurellari@lincoln.ac.uk,m.ghogho@ieee.org
}

\begin{abstract}
We consider the issue of designing closed 3D UAV trajectories that allow for an energy efficient collection of data with a UAV-aided wireless sensor network. We consider a 3D wireless channel model and a realistic dynamical model for the UAV. The proposed trajectory is largely derived analytically, thus making its online calculation computationally tractable. We also show the importance of using realistic dynamical and energy models for the UAV in designing efficient trajectories. This is done mainly by showing that minimising the flying time of the UAV is not equivalent to minimising its energy consumption. Simulation results corroborate these findings.
\end{abstract}

Index Terms- Wireless sensor network, UAV, data collection, trajectory planning.

\section{INTRODUCTION}

Wireless sensor networks (WSNs), composed of multiple coordinated low-cost sensor nodes (SNs) and a fusion center (FC), are considered as one of the most important emerging digital technologies. Their deployment has been witnessed in several sectors in the last 12 years such as environment monitoring, building infrastructure, agriculture, health-care and military applications [2-8] to name but just a few. There are a number of different strategies as to how the data from each $\mathrm{SN}$ will be efficiently collected/delivered. In general, the FC may be a static node (i.e., with no movement capabilities) or a dynamic node (e.g., Unmanned Aerial Vehicles (UAVs)).

Recently, there has been an increasing interest in the exploitation of mobility and UAVs [9] to improve the performance of WSNs, e.g. routing in [10], energy balancing in [11], energy consumption and operational lifetime [12], and improving security [13]. UAVs can also be used to collect data from SNs [14-16]. This is a suitable solution to reduce the energy consumption of a large WSN when the SNs are located far from the FC.

In the context of trajectory design of UAV-enabled data collection in WSNs, most of the existing work consider a twodimensional (2D) UAV trajectory (e.g., [9, 14, 15]). Joint op-

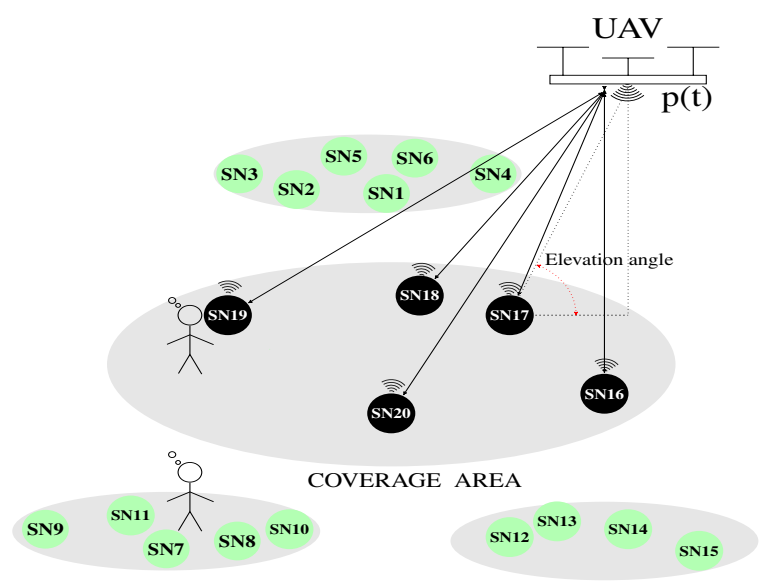

Fig. 1. Schematic communication architecture of the studied UAV-aided WSN. SNs represented with a black/green color are active/non-active.

timization of SNs' wake-up schedule and UAVs' trajectory is considered in [14], while [15] consider the design of the UAV's trajectory so that the mean square error is minimized. Recent work [16] explores the design of the 3D UAV trajectory to maximize the minimum average data collection rate in Rician fading channels. In this paper, we consider a UAVenabled WSN composed of a number of sensor nodes (SNs). The SNs are put in sleeping mode for energy saving and are scheduled to transmit data only when a UAV wake-up signal is broadcasted (e.g., beacon signal). Similar to [16], we design the 3D trajectory of the UAV and propose a low cost but energy efficient solution for the trajectory design problem. We consider a realistic dynamical model for the UAV, as opposed to previous papers considering similar problems, and show the necessity of using such realistic UAV models when dealing with this type of problems. We also consider a realistic air-to-ground communications channel. To the authors' knowledge, this is the first time that a 3D UAV trajectory is optimised in this context while considering a realistic dynamical model for the UAV. 


\section{SYSTEM MODEL}

In this paper, we consider a UAV-aided WSN, composed of $J$ SNs and a UAV. The position of the $j$ th SN and UAV are denoted by $\mathbf{c}_{j}=\left[x_{j}, y_{j}, 0\right]^{\mathrm{T}}$ and $\mathbf{p}(t)=[x(t), y(t), z(t)]^{\mathrm{T}}$ respectively, where $z(t)$ represents the altitude and $\mathcal{C}=\left\{\mathbf{c}_{j}\right\}_{j=1}^{J}$.

Next, we discuss the communication network model.

\subsection{Communications Channel Model}

We use a 3D channel model as in $[14,17,18]$. The channel power loss between the $j$ th SN and the UAV for the single frequency narrowband signal is shown to be:

$$
L_{d B}\left(\mathbf{c}_{j}, \mathbf{p}(t) \mid \eta_{j}\right)=20 \log \left(\left\|\mathbf{c}_{j}-\mathbf{p}(t)\right\|_{2}\right)+\eta_{j}
$$

where $\eta_{j}$ is a random variable representing the excessive path loss [19] that takes the value of $\eta_{L}$ if there is a line of sight (LoS) and $\eta_{N}$ otherwise. The LoS probability between the $j$ th $\mathrm{SN}$ and the UAV is given by [14]:

$$
\mathrm{P}_{\mathrm{j}}^{\mathrm{L}}(\mathbf{p}(t))=\left(1+a \exp \left[-b \arctan \left(\frac{\Delta_{j}^{z}(\mathbf{p}(t))}{\Delta_{j}^{h}(\mathbf{p}(t))}\right)-a\right]\right)^{-1}
$$

where $\Delta_{j}^{z}(\mathbf{p}(t))$ and $\Delta_{j}^{h}(\mathbf{p}(t))$ are the vertical and horizontal distances, respectively, between the $j$ th SN and the UAV at time instant $t ; a$ and $b$ are environment specific coefficients.

The UAV is equipped with a single directional antenna pointing downwards whose directivity is assumed here to be $D\left(\psi_{j}(\mathbf{p}(t))\right)=\sin ^{2}\left(\psi_{j}(\mathbf{p}(t))\right)$ where $\psi_{j}(\mathbf{p}(t)) \in\left[0, \frac{\pi}{2}\right]$ is the elevation angle w.r.t. $j$ th SN (see Fig. 1). We assume that each SN has a single isotropic antenna and so the signal-tonoise ratio (SNR) at the UAV's receiver is given by:

$$
\Gamma^{j}\left(\mathbf{p}(t) \mid \eta_{j}\right)=\gamma_{0} D\left(\psi_{j}(\mathbf{p}(t))\right) / L\left(\mathbf{c}_{j}, \mathbf{p}(t) \mid \eta_{j}\right)
$$

where $\gamma_{0}$ represents the average transmission power to UAV's receiver noise power ratio.

\subsection{Communications Network Model}

The UAV is tasked to periodically collect data from the WSN by using a 3D trajectory passing through $K$ stopping points (STPs) $\left\{\mathbf{s}_{k}\right\}_{k=1}^{K}$, which are calculated at the beginning of each tour. The UAV will hover at each STP during data collection. The tour starts and finishes at a control center (CC) (located at $\mathbf{s}_{0}$ ), where the UAV delivers the data, and also recharges batteries when depleted. Note that the UAV will visit the stopping points $\left\{\mathbf{s}_{k}\right\}_{k=1}^{K}$ in a predefined order.

For every tour, each $\mathrm{SN}$ generates $B$ bits. If during one tour, a SN is not activated to transmit its data to the UAV (because of not being located in any of the UAV's coverage areas during the tour), then this data is lost. Once enabled to transmit to the UAV, the SN forwards the data using packets of fixed payload length $h$, with header length $L$, and without re-transmission mechanisms (i.e., data received with errors is discarded). The transmission time for the $j$ th $\mathrm{SN}$, neglecting the time guard between packets, is $\tau_{t x}=N_{P} N_{s} T_{s}, \forall j$, where $N_{p}=B / h\left(N_{s}=(h+L) / \log _{2}(M)\right)$ represents the number of packets transmitted (symbols per packet); $T_{s}$ is the symbol duration and $M$ is the modulation order. We consider the M-PSK modulation scheme. The probability that the UAV (located at the STP $\mathbf{s}_{k}$ ), receives an error - free packet from the $j$ th $\mathrm{SN}$, conditioned on $\eta_{j}$, can then be expressed as:

$$
\mathrm{P}_{\mathrm{j}}^{\mathrm{efp}}\left(\mathbf{s}_{k} \mid \eta_{j}\right)=\left(1-2 Q\left(\sin \left(\frac{\pi}{M}\right) \sqrt{2 \Gamma_{j}\left(\mathbf{s}_{k} \mid \eta_{j}\right)}\right)\right)^{N_{s}}
$$

where $Q(\cdot)$ is the Q-function. It is not difficult to show that the number of packets successfully received by the UAV from the $j$ th $\mathrm{SN}$ is a binomial random variable with mean $\mu_{j}\left(\mathbf{s}_{k}\right)=N_{p} \mathbb{E}\left[\mathrm{P}_{\mathrm{j}}^{\text {efp }}\left(\mathbf{s}_{k} \mid \eta_{j}\right)\right]$ where the expected value, taken w.r.t. $\eta_{j}$, can be calculated using (2).

Now, during one tour, the $j$ th SN will be enabled to transmit to the UAV if and only if the received SNR is higher than some threshold $\gamma$ (related to the UAV's sensitivity). If the $j$ th SN observes an SNR superior to $\gamma$ at various different STPs of the UAV then the $j$ th SN will transmit to the UAV only when it is located at the STP that experiences the highest SNR. We define the set of SNs associated to $\mathbf{s}_{k}$ as $\mathcal{C}_{k}$. Finally, if the cardinality of $\mathcal{C}_{k},\left|\mathcal{C}_{k}\right|>1$, then the SNs belonging to $\mathcal{C}_{k}$ will use TDMA for transmission.

\subsection{Drone's Dynamical Model}

In this paper, we consider the UAV to be a quadrotor. For mathematical simplicity, we neglect the inertia of the motors (but still consider the UAV rotational inertia), force the yaw angle to zero and restrict both the roll $\phi(t)$ and the pitch $\theta(t)$ angles to small values. The above assumptions will yield the following simplified dynamical model (DM):

$$
\begin{aligned}
\ddot{\mathbf{p}}(t) & =[\theta(t),-\phi(t), 1]^{\mathrm{T}} u_{z}(t) / m-[0,0, g]^{\mathrm{T}} \\
{[\ddot{\phi}(t), \ddot{\theta}(t)] } & =\left[u_{y}(t), u_{x}(t)\right] \ell / I
\end{aligned}
$$

where $m$ is the total UAV's mass, $\ell$ is the length from the UAV's center to the rotors, $I$ is the UAV rotational inertia and $g$ is the gravitational constant. We define the control signal $\mathbf{u}(t)=\left[u_{x}(t), u_{y}(t), u_{z}(t)\right]^{\mathrm{T}}$. Finally, we assume the UAV energy consumption to be $E_{m}\left(t_{0}, t_{1}\right)=\int_{t_{0}}^{t_{1}}\|\mathbf{u}(\tau)\|_{2} \mathrm{~d} \tau$.

Next, we present our UAV trajectory planning solution.

\section{PROBLEM STATEMENT AND SOLUTION}

An energy-efficient 3D UAV trajectory for the data collection problem at hand may, given an energy budget, be formulated 
as a solution to the following optimisation problem:

$$
\begin{aligned}
& \max _{K, \mathbf{u}, \mathbf{s}_{k}, T} \frac{h \sum_{k=1}^{K} \sum_{\mathbf{c}_{j} \in \mathcal{C}_{k}} \mu_{j}\left(\mathbf{s}_{k}\right)}{E_{m}(0, T)}-\exp \left(\lambda\left(E_{m}(0, T)-E_{M}\right)\right) \\
& \text { s.t. } \\
& \mathbf{p}(t)=\mathbf{s}_{k}, \forall t \in\left[t_{k}, t_{k}+\left|\mathcal{C}_{k}\right| \tau_{t x}\right], k=1, \cdots, K \\
& \mathbf{p}(0)=\mathbf{p}(T)=\mathbf{s}_{0}, \operatorname{DM}(5), t_{k}<t_{k+1}
\end{aligned}
$$

The above optimization target is composed of the UAV's efficiency (i.e., the expected number of bits collected from the WSN divided by the energy spent) and a penalisation term associated with the UAV energy consumption ${ }^{1}$. The constraints ensure that the UAV: (i) passes through all the $K$ stopping points in predefined order; (ii) hovers at each stopping point until all the corresponding SNs have transmitted their data; (iii) starts and finishes at the CC; and (iv) obeys the DM (5).

Due to its complexity, obtaining an analytical solution to (6) is difficult if not impossible. So, next we propose a suboptimal but efficient solution.

\subsection{Suboptimal solution}

The DM (5) is nonlinear w.r.t. control signal $\mathbf{u}(t)$. Let us denote the time required for UAV to move from the STP $\mathbf{s}_{k-1}$ to the STP $\mathbf{s}_{k}$ as $\tau(k)$. For mathematical simplicity, this movement will be executed in two separate movements: a vertical and a horizontal movements of duration $\tau_{H}(k)$ and $\tau_{V}(k)$ respectively (i.e., $\tau(k)=\tau_{H}(k)+\tau_{V}(k)$ ). Clearly, (5) becomes now linear w.r.t. $\mathbf{u}(t)$ for a given (vertical/horizontal) movement. This allows us to optimize analytically $\mathbf{u}(t)$ using calculus of variations [26] to minimize the energy consumption. So, during the horizontal movement $\left(t \in\left[0, \tau_{H}(k)\right]\right)$, it can be shown that $u_{z}(t)=m g$; $u_{i}(t)=\left(\frac{-16800 t^{3}}{\tau_{H}^{7}(k)}+\frac{25200 t^{2}}{\tau_{H}^{6}(k)}-\frac{10080 t}{\tau_{H}^{5}(k)}+\frac{840}{\tau_{H}^{4}(k)}\right) \frac{I \Delta_{i}(k)}{g \ell}$ for $i=x, y$; with $\left[\Delta_{x}(k),-\Delta_{y}(k), \Delta_{z}(k)\right]^{\mathrm{T}}=\mathbf{s}_{k}-\mathbf{s}_{k-1}$. Similarly, during the vertical movement $\left(t \in\left[0, \tau_{V}(k)\right]\right)$, it can be shown that $u_{i}(t)=0, i=x, y ; u_{z}(t)=\frac{6 \Delta_{z}(k) m\left(\tau_{V}(k)-2 t\right)}{\tau_{V}^{3}(k)}+m g$.

Now, let us re-write $\tau_{H}(k)=T_{H} \beta_{k}\left(\tau_{V}(k)=T_{V} \alpha_{k}\right)$ where $T_{H}\left(T_{V}\right)$ is the total time spent in horizontal (vertical) movements required for a complete UAV tour, and $\left\{\beta_{k}\right\}_{k=0}^{K}$ $\left(\left\{\alpha_{k}\right\}_{k=0}^{K}\right)$ is a set of coefficients to be optimized (see later) that satisfy $\sum_{k=0}^{K}\left\{\alpha_{k}\right\}=1, \sum_{k=0}^{K}\left\{\beta_{k}\right\}=1, \alpha_{k} \geq 0$ and $\beta_{k} \geq 0$. The total amount of energy spent in horizontal and vertical movements can be shown to be:

$$
\begin{aligned}
& E_{H}=\left(\frac{100800 I^{2}}{g^{2} \ell^{2} T_{H}^{7}}\right) \sum_{k=0}^{K} \frac{\Delta_{H}^{2}(k)}{\beta_{k}^{7}}+(m g)^{2} T_{H} \\
& E_{V}=\frac{12 m^{2}}{T_{V}^{3}} \sum_{k=0}^{K} \frac{\Delta_{z}^{2}(k)}{\alpha_{k}^{3}}+(m g)^{2} T_{V}
\end{aligned}
$$

where $\Delta_{H}^{2}(k)=\Delta_{x}^{2}(k)+\Delta_{y}^{2}(k)$. First, we would like to find the optimum coefficients $\left\{\beta_{k}^{*}\right\}_{k=0}^{K}$ and $\left\{\alpha_{k}^{*}\right\}_{k=0}^{K}$. This

\footnotetext{
${ }^{1}$ The design parameter $\lambda$ must be a large positive number. This penalization term is negligible as long as $E_{m}(0, T)<E_{M}$.
}

is a complicated problem to solve directly (i.e., minimizing $\sum_{k=0}^{K} \frac{\Delta_{H}^{2}(k)}{\beta_{k}^{7}}$ subject to $\sum_{k=0}^{K} \beta_{k}=1$ and $\left.\beta_{k} \geq 0\right)$. So, we first consider the continuous version of this problem that can be efficiently solved analytically using calculus of variations and solving the corresponding Euler-Lagrange equations [26]. The corresponding continuous problem is to find the optimum function $f(v)$ that minimizes $\int_{0}^{T} \frac{g(v)}{f^{n}(v)} \mathrm{d} v$ subject to $n \in \mathbb{N}, \int_{0}^{T} f(v) \mathrm{d} v=1$, and $f(v) \geq 0$ for $v \in[0, T]$. The optimum solution $\left(f^{*}(v)\right)$ can be easily shown to be $f^{*}(v)=g(v)^{1 /(n+1)} / \int_{0}^{T} g(v)^{1 /(n+1)} \mathrm{d} v$. Both problems can be shown to be equivalent (the continuous and discrete versions); so, we then translate this result to $\left\{\beta_{k}\right\}_{k=0}^{K}$ and $\left\{\alpha_{k}\right\}_{k=0}^{K}$ which gives:

$$
\begin{aligned}
& \beta_{k}^{*}=\Delta_{H}^{1 / 4}(k)\left(\sum_{k=0}^{K} \Delta_{H}^{1 / 4}(k)\right)^{-1} \\
& \alpha_{k}^{*}=\Delta_{z}^{1 / 2}(k)\left(\sum_{k=0}^{K} \Delta_{z}^{1 / 2}(k)\right)^{-1} .
\end{aligned}
$$

Substituting (8) into (7) gives:

$$
\begin{aligned}
& \bar{E}_{H}=\left(\frac{100800 I^{2}}{g^{2} \ell^{2} T_{H}^{7}}\right)\left(\sum_{k=0}^{K} \Delta_{H}^{1 / 4}(k)\right)^{8}+(m g)^{2} T_{H} \\
& \bar{E}_{V}=\frac{12 m^{2}}{T_{V}^{3}}\left(\sum_{k=0}^{K} \Delta_{z}^{1 / 2}(k)\right)^{4}+(m g)^{2} T_{V} .
\end{aligned}
$$

Then, we optimize (9) w.r.t. $T_{V}$ and $T_{H}$ (i.e., by differentiating (9) w.r.t. $T_{V}$ and $T_{H}$ and equating to zero). This provides a full analytical description of the sub-optimal trajectory as a function of $\left\{\mathbf{s}_{k}\right\}_{k=1}^{K}$. The optimization of the STPs is complex and hence we propose a suboptimal approach to determine their horizonal positions using the $k$-means algorithm [29]. Indeed, we apply the latter on the set of SNs $\mathcal{C}$, with $K$ clusters, and set the horizontal positions of the unordered set of STPs to be those of the centroids of the $K$ clusters.

The STPs' altitude that maximizes the coverage area can be shown to be $z_{M}=\sqrt{\left(\frac{5 \gamma_{0}}{2 \gamma}\right)\left(\frac{P_{L}}{\eta_{L}}+\frac{1-P_{L}}{\eta_{N}}\right)}$ with $P_{L}=(1+a \exp [-b \pi / 2-a])^{-1}$ and let $z_{m}(k)$ be minimum $k$ th STP altitude that maintains full coverage ${ }^{2}$ of the SNs $\mathcal{C}_{k}$. Now, high altitudes have high LoS probability, but the distance to the receiver is large so the SNR might be low. On the other hand low altitudes have shorter distances to the receiver but also lower LoS probability hence also tend to have low SNR. Therefore for the $k$ th STP's altitude we propose two options: $z_{k}=\left(z_{m}(k)+z_{M}\right) / 2$ and $z_{k}=\max _{k}\left(z_{m}(k)\right)$.

Now that we have the 3D locations of the unordered STPs, we order them using the closest neighbor heuristics [28], which provides a sub - optimum but efficient solution to the Travel Salesman Problem (TSP). For simplicity, the cost assigned to each segment between stopping points is taken to be the Euclidean distance between the points. Then, this process is repeated for all values of $K$ up to a certain design limit $K_{M}$.

\footnotetext{
${ }^{2}$ Easily calculated numerically
} 
It is worth pointing out that the proposed sub-optimal but computationally efficient trajectory can be easily calculated online by the UAV using its limited on-board processing capabilities.

\subsection{Key Considerations and Discussions}

First, we note that the UAV energy consumption is a concave function of the time spent in the translation movements, see (9). So, the energy consumed becomes large if the time spent in translation movements becomes too short. Hence, the common strategy (applied in similar problems in the communications and signal processing communities) of considering the UAV flying time minimisation problem equivalent to its energy consumption minimisation [30] is in general wrong and can lead to a considerable reduction of the whole system's efficiency. This clearly demonstrates the need to consider more elaborated and realistic models for UAVs when studying this type of problems involving communications and robotics.

Second, the proposed solution does not ensure that all the SNs will be served by the UAV. If the UAV follows the same trajectory in every tour, then there will be a set of SNs that will be systematically ignored. This can be damaging as the data recorded by those SNs might be essential. To solve this, at the beginning of each tour, the UAV can randomly exclude some of the SNs that have already been served previously when calculating the new trajectory. This will increase the probability of being served for the previously ignored SNs.

Third, as mentioned in section 2.3, the UAV model considered is valid only for small roll and pitch angles, $\phi(t)$ and $\theta(t)$. But the solution proposed does not ensure this. So, if the solution obtained provides large values for those angles then some postprocessing can be done to arrange this, e.g., increasing $T_{H}$. This is an issue that will be addressed in future research.

\section{SIMULATION RESULTS}

In this section we present some simulation results. We select a WSN of $J=120 \mathrm{SN}$ distributed randomly on a square region of $140 \mathrm{~m} \times 140 \mathrm{~m}$. We set $M=4, h=16, L=8$, $T_{s}=100 \mathrm{~ms}, \gamma_{0}=1000, \gamma=1$. For the UAV we select $m=1.3 \mathrm{~kg}$ and $\ell / I=2.13$ corresponding to the values presented in [31]. For the 3D wireless channel model we select the values corresponding to the dense-urban scenario [18]. In Fig. 2 we present four different cases for the altitude of the STPs in the suboptimum UAV trajectory: (I) $z_{k}=\left(z_{m}(k)+z_{M}\right) / 2$; (II) $z_{k}=\max _{k}\left(z_{m}(k)\right)$; (III) $z_{k}=z_{M}$; (IV) $z_{k}=z_{m}(k)$. As long as $z_{k} \in\left[z_{m}(k), z_{M}\right]$, as in the four cases considered, the particular choice for the altitude of the STPs does not affect the percentage of covered SNs. This is why we only observe one curve for the coverage in Fig. 2.

If the energy limit $E_{M}$ in (6) is sufficiently high then the case (II) provides the best performance, followed by case (I),
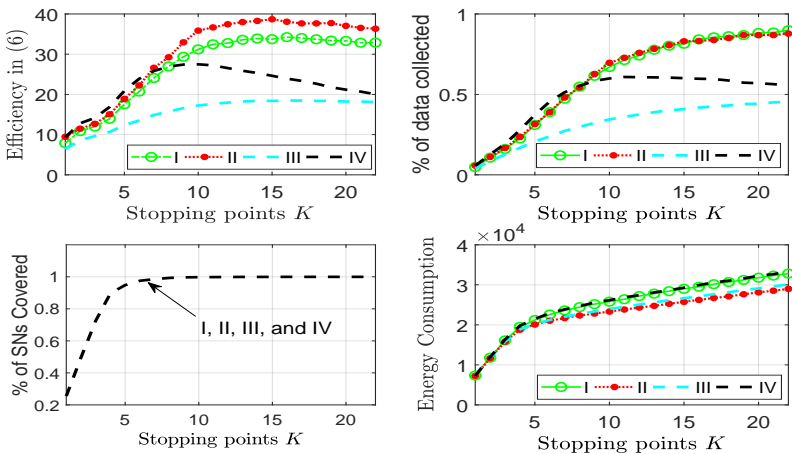

Fig. 2. Average performance of the proposed UAV trajectory vs. stopping points $(K)$ and for different altitude configurations.

for $K=15$ STPs providing coverage to $100 \%$ of the SNs but collecting $83 \%$ of the data generated. Though these two cases have similar performance in terms of data collection, case (II) provides a significantly lower energy consumption. This is due to the fact that the configuration of the STPs is planar (i.e., all the altitudes of the STPS are the same), and hence the amount of energy spent in vertical movements is minimised. This would suggest that, on average, as long as the altitude of the STPs is around the middle of the interval $\left[z_{\min }(k), z_{\max }\right]$ the performance in terms of data collection is relatively insensitive to the particular choice of $z_{k}$. Nevertheless, this is not a conclusive result and more research needs to be done regarding the choice of the altitudes. Specifically one question that needs to be answered is whether a planar configuration of the STPs would result in a more energy efficient trajectory than a 3D configuration of the STPs (i.e., where not all the STPs lie in the same horizontal plane).

Also note that if the energy limit $E_{M}$ in (6) is low, then the most efficient configuration is (IV). This shows that the optimum trajectory not only depends on the network characteristics but also on the battery of the UAV (which determines not only $E_{M}$ but also the mass $m$ of the UAV) and its DM, hence demonstrating the importance of using realistic dynamical models of UAVs in this type of problems.

\section{CONCLUSIONS}

In this paper we have presented a suboptimum trajectory that allows a UAV to collect data from a WSN in an energy efficient manner. We have derived analytical expressions for the trajectory as a function of the STPs. We have also shown the importance of considering realistic UAV models in this type of problems. In particular, we have shown that: i) minimizing the flying time of the UAV is not equivalent to minimizing its energy consumption, and ii) the optimum configuration of the STPs depends on the energy limit of the UAV. The comparison of planar and 3D trajectories in terms of energy efficiency needs further investigation. This will be addressed in future research. 


\section{REFERENCES}

[1] G. Kortuem, F. Kawsar, V. Sundramoorthy, and D. Fitton, "Smart objects as building blocks for the Internet of things," in IEEE Internet Computing, vol. 14, no. 1, pp. 44-51, Feb. 2010.

[2] E. Nurellari, D. McLernon, and M. Ghogho, "Distributed Two-Step Quantized Fusion Rules via Consensus Algorithm for Distributed Detection in Wireless Sensor Networks," in IEEE Transactions on Signal and Information Processing over Networks, vol. 2, no. 3, pp. 321-335, Sept. 2016.

[3] B. Sadler, "Fundamentals of energy-constrained sensor network systems," in IEEE Aerospace and Electronic Systems Magazine, vol. 20, no. 8, pp. 17-35, Aug. 2005.

[4] E. Nurellari, D. McLernon, and M. Ghogho, "A Secure Optimum Distributed Detection Scheme in Under-Attack Wireless Sensor Networks," in IEEE Transactions on Signal and Information Processing over Networks, vol. 4, no. 2 , pp. 325-337, Jun. 2018.

[5] I. F. Akyildiz, W. Su, Y. Sankarasubramaniam, and E. Cayirci, "A survey on sensor networks," in IEEE Communication Magazine, pp. 102-114, Aug. 2002.

[6] E. Nurellari, D. McLernon, M. Ghogho, and S. Aldalahmeh, "Distributed Binary Event Detection Under Data-Falsification and EnergyBandwidth Limitation," in IEEE Sensors Journal, vol. 16, no. 16, pp. 6298-6309, Aug. 2016.

[7] G. J. Pottie and W. J. Kaiser, "Wireless integrated network sensors," in Communications of the ACM, vol. 43, no. 5, pp. 51-58, May 2000.

[8] E. Nurellari, S. Aldalahmeh, M. Ghogho, and D. McLernon, "Quantized Fusion Rules for Energy-Based Distributed Detection in Wireless Sensor Networks," in Proc. Sensor Signal Processing for Defence (SSPD), Edinburgh, Scotland, pp. 1-5, 8-9 Sept. 2014.

[9] Y. Zeng, R. Zhang, and T. J. Lim, "Wireless communications with unmanned aerial vehicles: opportunities and challenges," in IEEE Communications Magazine, vol. 54, issue. 5 , pp. 36-42, May. 2016.

[10] A. Vahdat and D. Becker, "Epidemic routing for partially-connected ad hoc networks," Duke University, Tech. Rep., 2000.

[11] D. Bonilla Licea, E. Nurellari, and M. Ghogho, "Energy balancing for robotic aided clustered wireless sensor networks using mobility diversity algorithms," in 26th European Signal Processing Conference (EUSIPCO), pp. 1829-1833, Rome, Sep. 2018.

[12] E. Nurellari, D. Bonilla Licea, and M. Ghogho, "Optimum Trajectory Planning for Robotic Data Ferries in Delay Tolerant Wireless Sensor Networks," in 27th European Signal Processing Conference (EUSIPCO), pp., La Coruna, Spain, Sep. 2019.

[13] D, Bonilla Licea, E. Nurellari, and M. Ghogho, "Optimum Distributed Intruder's Detection in Wireless Mobile Sensor Networks," submitted in IEEE Transactions on Signal and Information Processing over Networks.

[14] C. Zhan, Y, Zeng, and R. Zhang, "Energy-Efficient Data Collection in UAV Enabled Wireless Sensor Network," in IEEE Wireless Communications Letters, vol. 7, issue. 3, pp. 328-331, June 2018.

[15] C. Zhan, Y, Zeng, and R. Zhang, "Trajectory Design for Distributed Estimation in UAV-Enabled Wireless Sensor Network," in IEEE Transactions on Vehicular Technology, vol. 77, no. 10, pp. 1015510159, October 2018

[16] C. You, Y, Zeng, and R. Zhang, "3D Trajectory Optimization in Rician Fading for UAV-Enabled Data Harvesting," in IEEE Transactions on Wireless Communications, vol. 18, no. 6, pp. 3192-3207, June 2019.
[17] M. Alzenad, A. El-Keyi, and H. Yanikomeroglu, "3D Placement of an Unmanned Aerial Vehicle Base Station for Maximum Coverage of Users with Different QoS Requirements", in IEEE 18th International Conference on Communication Technology (ICCT), October 2018.

[18] R. Irem Bor Yaliniz, A. El-Keyi, and H. Yanikomeroglu, "Efficient 3-D Placement of an Aerial Base Station in Next Generation Cellular Networks", in IEEE International Conference on Communications (ICC), July 2016.

[19] A. Al-Hourani, S. Kandeepan, and S. Lardner, "Optimal LAP altitude for maximum coverage", IEEE Wireless Commununications Letters, vol. 3 , no. 6 , pp. $569-572$, Dec. 2014

[20] Marvin K. Simon and Mohamed-Slim Alouini, Digital Communication over Fading Channels, John Wiley \& Sons. Inc, 2005.

[21] D. Bonilla Licea, D. McLernon and M. Ghogho, "Mobile Robot Path Planners With Memory for Mobility Diversity Algorithms", IEEE Transactions on Robotics, Vol. 33, Iss 3, April. 2017.

[22] M. Gudmundson, "Correlation model for shadow fading in mobile radio systems," Electron. Lett., vol. 27, Nov. 1991.

[23] A. Goldsmith, Wireless Communications, Cambridge university press, 2005. 3, April 2017

[24] S. Russell, P. Norving, Artificial Intelligence: A Modern Approach, Prentice Hall, 2003.

[25] M. Malmirchegini and Y. Mostofi, "On the Spatial Predictability of Communication Channels", IEEE Transactions on Wireless Communications, Vol. 11, Iss. 3, March 2012, pp. 964 - 978.

[26] D. E. Kirk, Optimal Control Theory: An Introduction., New York, NY, USA: Dover, 2004.

[27] S. Russell and P. Norving, Artificial Intellingence: A Modern Approach. Upper Saddle River, NJ, USA: Prentice-Hall, 2003,

[28] Donald Davendra Traveling Salesman Problem, Theory and Applications, InTech,2010.

[29] Gareth James, Daniela Witten, Trevor Hastie and Robert Tibshirani, An Introduction to Statistical Learning with Applications in $R$, Springer, 2013.

[30] Osama M. Bushnaq, Abdulkadir Celik, Hesham ElSawy, MohamedSlim Alouini, and Tareq Y. Al-Naffouri, "Aeronautical Data Aggregation and Field Estimation in IoT Networks: Hovering \& Traveling Time Dilemma of UAVs", IEEE Transactions on Wireless Communications, vol. 18, iss. 10, October 2019.

[31] Fabio Morbidi, Roel Cano and David Lara,"Minimum-Energy Path Generation for a Quadrotor UAV", Proceedings of the 2016 IEEE International Conference on Robotics and Automation (ICRA), Stockholm, Sweden, May, 2016. 